\title{
Unveiling dipolar spectral regimes of large dielectric Mie spheres from helicity conservation
}

\author{
Jorge Olmos-Trigo $\odot,{ }^{1, *}$ Diego R. Abujetas $\odot,{ }^{1,2}$ Cristina Sanz-Fernández $\odot,{ }^{3}$ Xavier Zambrana-Puyalto $\odot,{ }^{4}$ Nuno de Sousa $\odot,{ }^{1}$ \\ José A. Sánchez-Gil $\odot,{ }^{2}$ and Juan José Sáenz ${ }^{1,5}$ \\ ${ }^{1}$ Donostia International Physics Center (DIPC), 20018 Donostia-San Sebastián, Spain \\ ${ }^{2}$ Instituto de Estructura de la Materia (IEM-CSIC), Consejo Superior de Investigaciones Científicas, Serrano 121, 28006 Madrid, Spain \\ ${ }^{3}$ Centro de Física de Materiales (CFM-MPC), Centro Mixto CSIC-UPV/EHU, 20018 Donostia-San Sebastián, Spain \\ ${ }^{4}$ Istituto Italiano di Tecnologia, Via Morego 30, 16163 Genova, Italy \\ ${ }^{5}$ IKERBASQUE, Basque Foundation for Science, 48013 Bilbao, Spain
}

(Received 28 March 2020; accepted 14 September 2020; published 5 October 2020; corrected 9 March 2022)

\begin{abstract}
Controlling the helicity and directionality of the scattered light by dielectric particles is paramount to a variety of phenomenology of interest in all-dielectric optics and photonics. In this paper, we demonstrate that the helicity of an incoming beam is not preserved when several multipoles contribute to the scattering. This inhibits the usual link between duality restoration and zero optical backscattering condition. In addition, we show that the helicity conservation in the scattering by high-refractive index dielectric Mie spheres can be used as a probe of pure-multipolar spectral regions, particularly of dipolar nature, beyond its presumed spectral interval. This finding reveals that the dipolar behavior is not necessarily limited to small particles, in contrast to the current understanding. Interestingly, our formalism allows us to demonstrate that the optimum forward light scattering condition, predicted for a particular nanosphere, is fulfilled for an infinite number of refractive indexes in these dipolar regimes.
\end{abstract}

DOI: 10.1103/PhysRevResearch.2.043021

In 1908, Gustav Mie presented his most relevant contribution to the electromagnetic (EM) theory by solving the scattering of a plane wave by a spherical particle [1]. As time passed by, the interest in what is nowadays referred to as Mie theory increased and was later extended to magnetic spheres presenting a nonzero relative permittivity $\epsilon$ and permeability $\mu$ [2]. In that work, Kerker et al. predicted a perfect zero optical backscattering condition, regardless of the size of the sphere, when the condition $\epsilon=\mu$ is satisfied. Thirty years later, the previous condition was linked to the restoration of a nongeometrical symmetry: EM duality [3]. Like all continuous symmetries, EM duality has a generator, and this was found by Calkin in 1965 [4]. Calkin showed that the conserved quantity related to the EM duality symmetry is the EM helicity $(\Lambda)$, defined as the projection of the total angular momentum onto the linear momentum of the light wave. However, there are no magnetic materials $(\mu \neq 1)$ at optical frequencies. Hence, the EM duality restoration together with its signatures (EM helicity conservation and absence of backscattered light) have not been experimentally verified.

In striking contrast, high-refractive index (HRI) dielectric nanospheres present strong magnetic and electric dipolar resonances in the visible [5] region, as well as in telecom and near-infrared frequencies [6]. Notably, when the

\footnotetext{
*jolmostrigo@gmail.com
}

Published by the American Physical Society under the terms of the Creative Commons Attribution 4.0 International license. Further distribution of this work must maintain attribution to the author(s) and the published article's title, journal citation, and DOI. electric and magnetic polarizabilities of the scatterer are identical, at the so-called first Kerker condition [7], the EM helicity is preserved [8-10] and the intensity in the backscattering direction is exactly zero [11-13]. These anomalous light scattering effects have been exploited in multiple branches of physics ranging from boosting light collection efficiency in plants [14], to dynamic control of cryptographic nanoprints [15], to maximizing the sensitivity of circular dichroism spectroscopy of chiral molecules [16-19]. The physical picture behind most of the aforementioned works consists of maximizing the efficiency of the scattered light while fulfilling the first Kerker condition. For a nanosphere object, the best candidate to fulfill the previous condition, referred to as optimum forward light scattering condition, was found to be a material with a refractive index of $m \approx 2.45$ in the limit of small particles [20,21]. Nonetheless, the ideal mapping from the scattering by magnetic spheres satisfying $\epsilon=\mu$ onto the scattering by larger dielectric spheres, beyond the dipolar regime, can only be achieved if and only if every pair of electric and magnetic Mie coefficients are identical, i.e., $a_{l}=b_{l} \forall l$, where $l$ is the multipole order.

In this paper, we demonstrate that the scattering of a sphere with $\epsilon=\mu$ cannot be exactly mimicked by a dielectric Mie sphere. This fact stems from a fundamental property of the Bessel functions, and it is general since it does not depend on the refractive index contrast, multipole order, incoming polarization, or ratio between the incident wavelength and particle size. Specifically, it implies that if $a_{j}=b_{j}$ then $a_{l} \neq b_{l}$ $\forall l \neq j$. Consequently, the EM helicity conservation and the zero optical backscattering condition cannot be simultaneously achieved in scattering processes in which several multipoles contribute to the optical response. Nevertheless, 
and as a result of our proof, we show that the conservation of the EM helicity $(\langle\Lambda\rangle \approx 1)$ implies the existence of puremultipolar spectral regions, ${ }^{1}$ specifically of dipolar nature. We show that these emerge beyond the magnetic and electric quadrupole resonances for HRI materials. This can be summarized as follows: the concept of small particle $[5,6]$ is sufficient, but not necessary, to assume a dipolar response. Additionally, we determine that the optimum forward light scattering condition arises for an infinite number of refractive indexes at a fixed ratio between the incident wavelength and particle size in these dipolar regimes, in contrast to previous interpretations [20,21].

Mie theory [1] gives the exact analytical solution of Maxwell's equations for a homogeneous spherical particle in a lossless homogeneous medium under plane wave illumination. The scattering efficiency can be written as [22]

$$
Q_{\text {sca }}=\frac{2}{x^{2}} \sum_{l=1}^{\infty}(2 l+1)\left(\left|a_{l}\right|^{2}+\left|b_{l}\right|^{2}\right),
$$

where $Q_{\text {sca }}=\sigma_{\text {sca }} / \pi R^{2}, \sigma_{\text {sca }}$ being the scattering cross section and $R$ the radius of the particle. Here, $x=k R$ is the size parameter, where $k=m_{\mathrm{h}} k_{0}=m_{\mathrm{h}}\left(2 \pi / \lambda_{0}\right), \lambda_{0}$ being the incoming wavelength in vacuum and $m_{\mathrm{h}}$ the refractive index of the external medium. The scattering properties depend on the refractive index contrast between the particle and the external medium, defined as $m=m_{\mathrm{p}} / m_{\mathrm{h}}$, where $m_{\mathrm{p}}$ is the refractive index of the particle. For nonmagnetic particles, the electric and magnetic Mie coefficients are given by [22]

$$
a_{l}=\frac{m S_{l}(m x) S_{l}^{\prime}(x)-S_{l}(x) S_{l}^{\prime}(m x)}{m S_{l}(m x) C_{l}^{\prime}(x)-C_{l}(x) S_{l}^{\prime}(m x)}
$$

and

$$
b_{l}=\frac{S_{l}(m x) S_{l}^{\prime}(x)-m S_{l}(x) S_{l}^{\prime}(m x)}{S_{l}(m x) C_{l}^{\prime}(x)-m C_{l}(x) S_{l}^{\prime}(m x)} .
$$

Here $S_{l}(z)=\sqrt{\frac{\pi z}{2}} J_{l+\frac{1}{2}}(z)$ and $C_{l}(z)=\sqrt{\frac{\pi z}{2}} H_{l+\frac{1}{2}}(z)$ denote the Riccati-Bessel functions, where $J_{l+\frac{1}{2}}(z)$ and $H_{l+\frac{1}{2}}(z)$ are the Bessel and Hankel functions, respectively.

In this notation, the condition $a_{l}(m, x)=b_{l}(m, x) \forall l$ would imply the restoration of the EM duality [8]. For each multipole order $l$, and according to Eqs. (2) and (3), this nonmagnetic generalized duality condition requires either $S_{l}(m x)=0$ or $S_{l}^{\prime}(m x)=0$ [22,23]. Generally, these solutions are embedded in

$$
a J_{l+\frac{1}{2}}(m x)+b m x J_{l+\frac{1}{2}}^{\prime}(m x)=0,
$$

where $a, b \in \mathbb{R}$. Notice that when $a=1$ and $b=0$ the node of the first kind emerges, i.e., $S_{l}(m x)=0$, while for $a=1$ and $b=2$ the node of the second kind arises, i.e., $S_{l}^{\prime}(m x)=0$. At this point, let us make use of the following lemma [24]:

(1) When $v>-1$ and $a, b \in \mathbb{R}$ such as $a^{2}+b^{2} \neq 0$, then no function of the type $a J_{v}(z)+b z J_{v}^{\prime}(z)=0$ can have a repeated zero other than $z=0$.

\footnotetext{
${ }^{1}$ We refer to as pure-multipolar scattering processes those that can be described with just one multipolar order $l$.
}

This lemma necessarily means that Eq. (4) cannot be formally satisfied $\forall l$ for a fixed size parameter $m x$. On physical grounds, this phenomenon implies that, when $a_{j}(m, x)=$ $b_{j}(m, x)$, no other pair of electric and magnetic Mie coefficients can be identical, $a_{l}(m, x) \neq b_{l}(m, x) \forall l \neq j$. That is, Lemma 1 analytically shows that the EM helicity cannot be totally conserved in scattering processes involving several multipoles for dielectric particles with $\mu=1$. This fact precludes the ideal restoration of the EM duality and its link with the perfect zero optical backscattering condition regardless of the multi-pole order, size parameter $x$, and refractive index contrast [3]. Nevertheless, the EM helicity can be conserved in lossless pure-multipolar spectral regions, specifically in dipolar spectral regimes, as we will shortly see. Let us explicitly calculate the expected value of the scattered EM helicity arising from a dielectric Mie sphere. For that, let us use the formulation of Mie theory in the helicity basis [25]. We expand the incoming beam electric field $\mathbf{E}_{\mathrm{inc}}$ in terms of vector spherical wave functions (VSWFs), $\Psi_{l m}^{\sigma}$, with well-defined helicity, $\sigma= \pm 1[26,27]$. In this basis,

$$
\mathbf{E}_{\mathrm{inc}}=\sum_{\sigma= \pm 1} \mathbf{E}_{\mathrm{inc}}^{\sigma}, \quad \mathbf{E}_{\mathrm{inc}}^{\sigma}=E_{0} \sum_{l=1}^{\infty} \sum_{m=-l}^{+l} C_{l m}^{\sigma} \boldsymbol{\Psi}_{l m}^{\sigma},
$$

where $\boldsymbol{\Psi}_{l m}^{\sigma}$ is defined as

$$
\begin{gathered}
\boldsymbol{\Psi}_{l m}^{\sigma}=\frac{1}{\sqrt{2}}\left[\boldsymbol{N}_{l m}+\sigma \boldsymbol{M}_{l m}\right], \\
\boldsymbol{M}_{l m} \equiv j_{l}(k r) \boldsymbol{X}_{l m}, \quad \boldsymbol{N}_{l m} \equiv \frac{1}{k} \nabla \times \boldsymbol{M}_{l m}, \\
\boldsymbol{X}_{l m} \equiv \frac{1}{\sqrt{l(l+1)}} \mathbf{L} Y_{l}^{m}(\theta, \varphi) .
\end{gathered}
$$

Here, $\boldsymbol{M}_{l m}$ and $\boldsymbol{N}_{l m}$ are Hansen's multipoles, $\boldsymbol{X}_{l m}$ denotes the vector spherical harmonic [28], $j_{l}(k r)$ are the spherical Bessel functions, $Y_{l}^{m}$ are the spherical harmonics, $C_{l m}^{\sigma}$ are the incident coefficients characterizing the nature of the incoming wave, and $\mathbf{L}=\{-i \mathbf{r} \times \nabla\}$ is the total angular momentum operator. Let us recall that the multipoles $\boldsymbol{\Psi}_{l m}^{\sigma}$ are simultaneous eigenvectors of $\mathbf{J}^{2}, J_{z}$ [28], and the helicity operator $\boldsymbol{\Lambda}$ [3], with eigenvalues $l(l+1), m$, and $\sigma$, respectively. Notice that the helicity operator can be expressed as $\boldsymbol{\Lambda}=(1 / k) \nabla \times$ for monochromatic fields. The scattered fields outside the sphere, $\mathbf{E}_{\text {sca }}=\mathbf{E}_{\text {sca }}^{+}+\mathbf{E}_{\text {sca }}^{-}$, can be written in terms of "outgoing" VSWFs, $\boldsymbol{\Phi}_{l m}^{\sigma^{\prime}}$, as

$$
\begin{aligned}
\mathbf{E}_{\mathrm{sca}}^{\sigma} & =E_{0} \sum_{l=1}^{\infty} \sum_{m=-l}^{+l} D_{l m}^{\sigma} \boldsymbol{\Phi}_{l m}^{\sigma}, \\
\left(\begin{array}{l}
D_{l m}^{+} \\
D_{l m}^{-}
\end{array}\right) & =-\left(\begin{array}{ll}
{\left[a_{l}+b_{l}\right]} & {\left[a_{l}-b_{l}\right]} \\
{\left[a_{l}-b_{l}\right]} & {\left[a_{l}+b_{l}\right]}
\end{array}\right)\left(\begin{array}{l}
C_{l m}^{+} \\
C_{l m}^{-}
\end{array}\right),
\end{aligned}
$$

where the "outgoing" VSWFs, $\boldsymbol{\Phi}_{l m}^{\sigma^{\prime}}$, are defined as in Eq. (6), with $j_{l}(k r)$ being replaced by the outgoing spherical Bessel functions $h_{l}(k r)$. In this framework, the expected value of the EM helicity of the scattered field is generally given by

$$
\langle\Lambda\rangle=\frac{\left\langle\boldsymbol{E}_{\mathrm{sca}}^{*} \cdot\left(\boldsymbol{\Lambda} \boldsymbol{E}_{\mathrm{sca}}\right)\right\rangle}{\left\langle\boldsymbol{E}_{\mathrm{sca}}^{*} \cdot \boldsymbol{E}_{\mathrm{sca}}\right\rangle}=\frac{\sum_{l=1}^{\infty} \sum_{m=-l}^{+l}\left[\left|D_{l m}^{+}\right|^{2}-\left|D_{l m}^{-}\right|^{2}\right]}{\sum_{l=1}^{\infty} \sum_{m=-l}^{+l}\left[\left|D_{l m}^{+}\right|^{2}+\left|D_{l m}^{-}\right|^{2}\right]} .
$$


Equation (10) is a general result showing the expected value of the EM helicity after scattering for an arbitrary incident beam. When the sphere is illuminated by a circularly polarized plane wave with helicity $\sigma= \pm 1$ [26], the scattered field is a combination of multipolar modes with fixed $m=\sigma$. As a result, it can be shown that

$$
\langle\Lambda\rangle=\sigma \frac{1-T}{1+T}, \quad \text { where } T=\frac{\sum_{l=1}^{\infty}\left|C_{l \sigma}^{\sigma}\right|^{2}\left|a_{l}-b_{l}\right|^{2}}{\sum_{l=1}^{\infty}\left|C_{l \sigma}^{\sigma}\right|^{2}\left|a_{l}+b_{l}\right|^{2}}
$$

is the helicity transfer function [25], and $C_{l \sigma}^{\sigma}=$ $\sigma i l \sqrt{4 \pi(2 l+1)}$ are the plane wave coefficients [28]. Now, when $T$ goes to zero, the scattered EM helicity is identical to the EM helicity of the incoming beam. Nonetheless, as we have previously demonstrated via Lemma 1, the EM helicity cannot be ideally conserved in scattering processes where several multipoles contribute to the optical response of the sphere. However, the EM helicity can be preserved in pure-multipolar spectral regions, particularly in dipolar regimes, as can be inferred from Fig. 1. First, we identify the dipolar regions (white color) from Fig. 1(a), where the percentage error of assuming a dipolar response, i.e., $Q_{\mathrm{error}}^{\mathrm{dip}}=\left|Q_{\mathrm{sca}}^{\mathrm{dip}} / Q_{\mathrm{sca}}-1\right| \%$, versus the $y=m x$ size parameter and refractive index contrast $m$ is depicted. Notice that $Q_{\text {sca }}^{\text {dip }}$ corresponds to Eq. (1) but retaining only $l=1$ (dipolar contribution). Surprisingly, several dipolar spectral regimes are found far beyond its presumed spectral interval [beyond the magnetic $\left(m_{\mathrm{q}}\right)$ and electric quadrupole $\left(e_{\mathrm{q}}\right)$ resonances] for HRI spheres with $m \geqslant 3.5$. Second, in Fig. 1(b) we show the expected value of the EM helicity after scattering, $\langle\Lambda\rangle$, under plane wave illumination with well-defined helicity, $\sigma=+1$ [see Eq. (11)]. As can be inferred from the attached color bar, $\langle\Lambda\rangle \approx 1$ when the $s_{1}^{\prime(q)}$ vertical trajectories, corresponding to the $q$ th zeros of $S_{1}^{\prime}(m x)=0$, pass through a dipolar spectral region. In contrast and as a result of Lemma $1,\langle\Lambda\rangle$ is not conserved when several multipoles contribute to the scattering. Notice that the emergence of dipolar spectral regions does not entail $\langle\Lambda\rangle \approx 1$ in the entire dipolar regime, which can be observed upon comparing the white regions of Fig. 1(a) with their corresponding regions of Fig. 1(b). Moreover, it is worth noticing that, for lossy spheres, the EM helicity cannot be preserved [23]. This physical picture remains valid for dissipating pure-multipolar regions such as dipolar regimes in the visible spectral range, e.g., a dielectric Ge nanosphere [23] or a Si nanosphere [29].

In order to get a deeper insight into the appearing of these dipolar spectral regimes, in Fig. 2 we analyze the dipolar contribution (blue dashed line) to the total scattering efficiency (black solid line) for two different dielectric-like spheres [30]: titanium oxide $\left(\mathrm{TiO}_{2}\right)$ with $m=2.5$ and germanium $(\mathrm{Ge})$ with $m=4$. While in the case of $\mathrm{TiO}_{2}$ the dipolar regime [blue background in Fig. 2(a)] just emerges in the limit of small particle [5,6], the scattering efficiency arising from the Ge sphere presents an unexpected dipolar regime beyond the point-dipole approximation in the interval given by $5.75<$ $y<6.25$ [narrow blue background in Fig. 2(b)], far beyond the $m_{q}$ and $e_{q}$ resonances. Let us recall that, in this dipolar regime, the EM helicity is preserved at $s_{1}^{\prime(2)}$ for the Ge sphere. This phenomenon implies that the zero optical backscattering condition can be fulfilled for dipolar HRI microspheres.

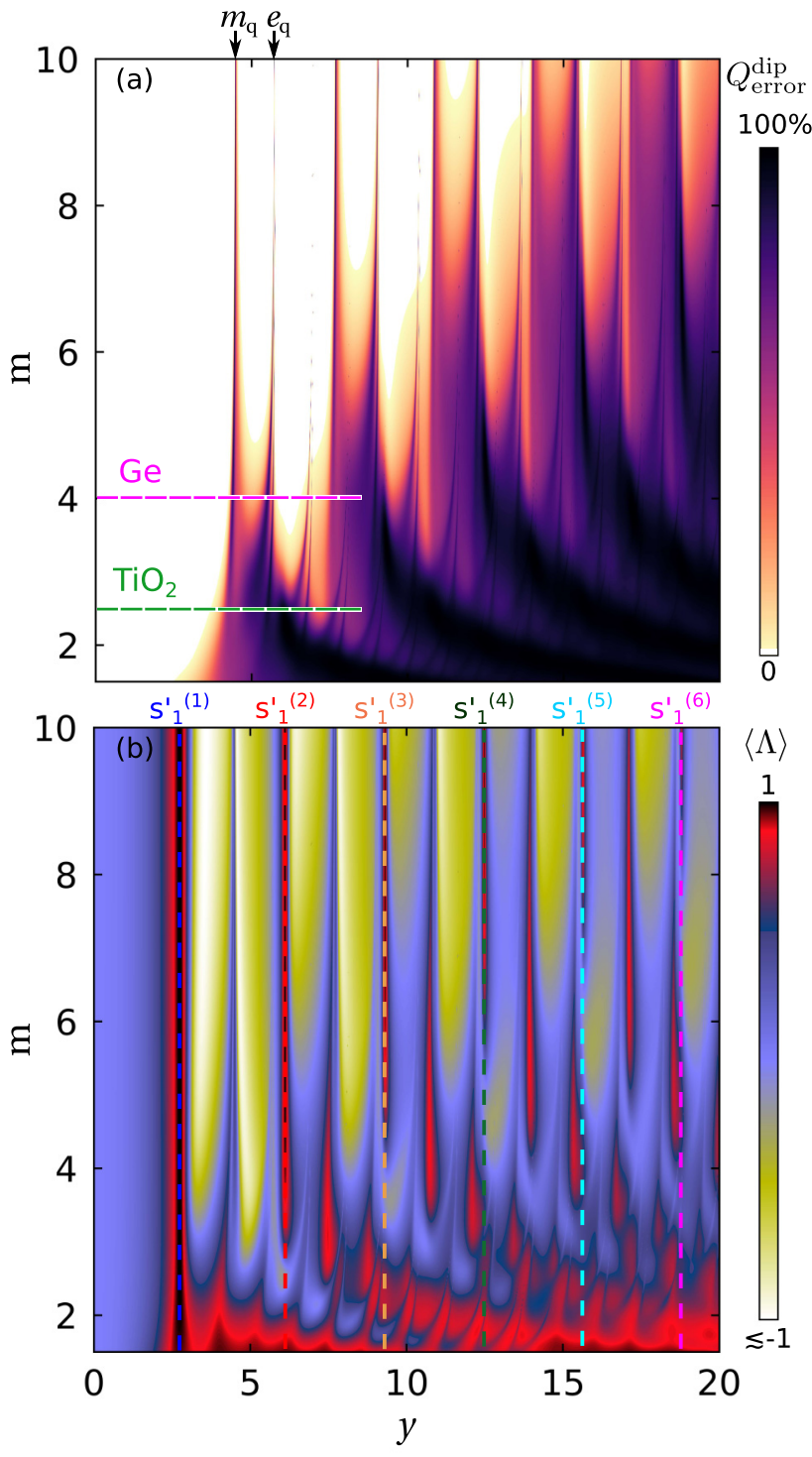

FIG. 1. (a) Percentage error of assuming a dipolar response, i.e., $Q_{\text {error }}^{\text {dip }}=\left|Q_{\text {sca }}^{\text {dip }} / Q_{\text {sca }}-1\right| \%$ vs the $y=m x$ size parameter and refractive index contrast $m$. The dipolar spectral regime corresponds to white regions. (b) Color map of the expected value of the EM helicity after scattering by a dielectric Mie sphere, $\langle\Lambda\rangle$, under well-defined EM helicity $(\sigma=+1)$ plane wave illumination.

Let us now briefly examine the optimum forward light scattering condition, predicted for a particular nanosphere with refractive index contrast $m \approx 2.45$ [20,21]. We address this problem by maximizing the dipolar scattering efficiency at the first Kerker condition given by the dipolar node of second kind, namely, $S_{1}^{\prime}(m x)=0,{ }^{2}$ which yields

$$
Q_{\mathrm{scat}}^{\max }=12\left|\frac{S_{1}^{\prime}\left(x_{\max }\right)}{x_{\max } C_{1}^{\prime}\left(x_{\max }\right)}\right|^{2} \approx 3.75, \quad \text { with } x_{\max } \approx 1.12 \text {. }
$$

\footnotetext{
${ }^{2}$ Notice that at the $S_{1}(m x)=0$ trajectories the scattering response cannot be described by dipolar modes.
} 


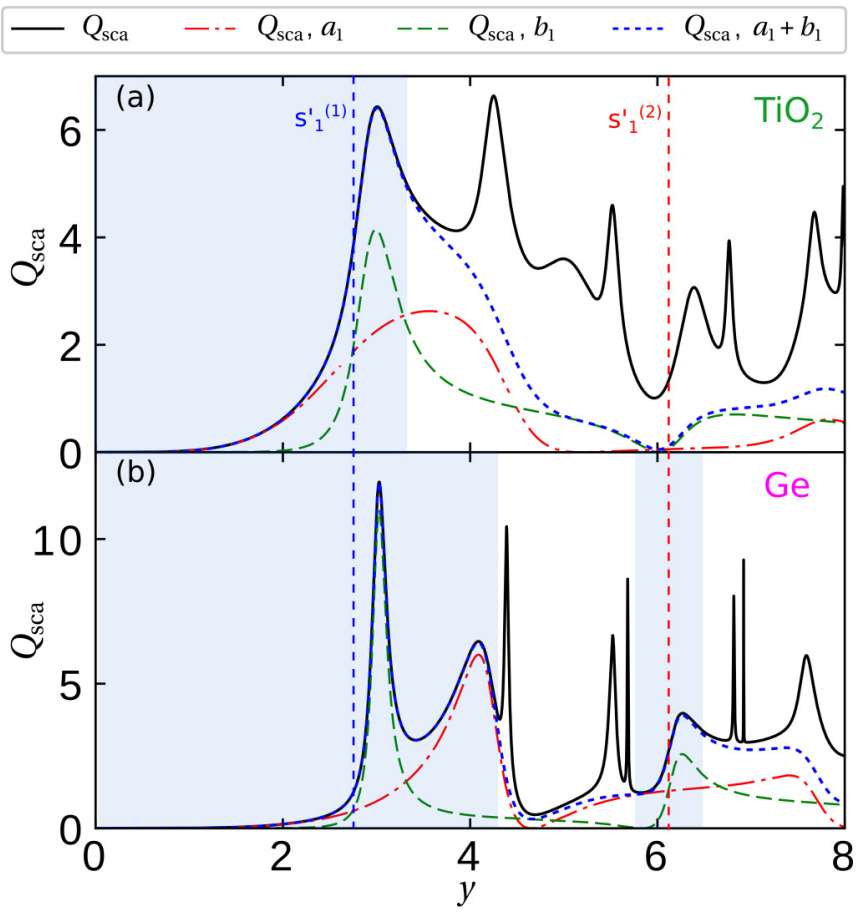

FIG. 2. Scattering efficiency from (a) $\mathrm{TiO}_{2}$-like sphere with $m=$ 2.5 and (b) Ge-like sphere with $m=4$. Blue regions illustrate the spectral regimes that are essentially described by a dipolar optical response. The first Kerker conditions are intentionally depicted in order to show that only $s_{1}^{\prime(1)}$ and $s_{1}^{(2)}$ lead to the conservation of the EM helicity for HRI spheres ( $m>3.5$ ), according to Fig. 1(b). The incoming beam is a circularly polarized plane wave with helicity $\sigma=+1$.

From $S_{1}^{\prime}(m x)=0$ evaluated at $x_{\max }$, namely, the size parameter that gives rise to the maximum scattering efficiency at the first Kerker condition, it is straightforward to derive the material(s) that satisfy the optimum forward light scattering condition. Mathematically, this is equivalent to finding the set of refractive contrast indexes satisfying $S_{1}^{\prime}\left(m x_{\max }\right)=0$, as shown graphically in Fig. 3(a). In Fig. 3(b), we show the scattering efficiency evaluated at the $x_{k}^{(q)}$, i.e., the size parameter that leads to spheres at the first Kerker condition, versus $m$.

To highlight the relevance of our results, we illustrate the scattering efficiency radiation pattern arising from nano-spheres satisfying the zero optical backscattering condition [12,13]. In Fig. 4(a) we illustrate the optimum forward light scattering condition normalized to its maximum value. In Fig 4(b) we show, for comparison, the zero optical backscattering condition arising from two seminal works $[12,13]$. In the latter, the intensity in the forward direction roughly represents $1 / 3$ of the optimum forward light scattering case.

In conclusion, we have unveiled a fundamental property of the Mie scattering of a dielectric sphere. Namely, when $a_{j}(m, x)=b_{j}(m, x)$, no other pair of electric and magnetic Mie coefficients can be identical, $a_{l}(m, x) \neq b_{l}(m, x) \forall l \neq j$. This finding precludes the connection between ideal EM duality restoration (or ideal helicity conservation) and the perfect zero optical backscattering condition in scattering processes where several multipoles contribute to the optical response of

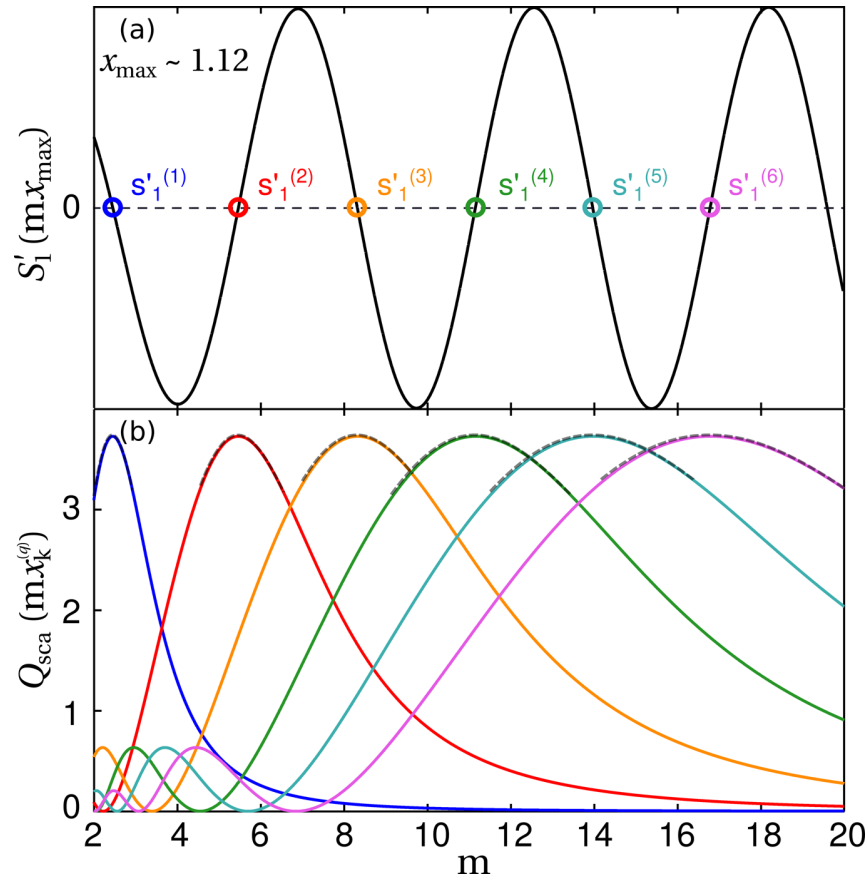

FIG. 3. (a) Node of the second kind, $S_{1}^{\prime}\left(m x_{\max }\right)$, as a function of the contrast index $m$. The $q$ th positive zero of this function, depicted by circles, satisfies the optimum forward light scattering condition. (b) Scattering efficiency, $Q_{\text {sca }}$, evaluated at $x_{\mathrm{k}}^{(q)}$, i.e., the size parameter that leads the first Kerker condition. The maximum value is given by $Q_{\text {sca }} \approx 3.75$. At these maxima, the full multipolar expansion (gray dashed lines) is essentially dipolar (solid lines).

an object [31]. The proof is general since it is solely based on a fundamental mathematical property of the Bessel functions and, thus, remains valid regardless of the particle size, refractive index contrast, incident wavelength, and multipole order. Nevertheless, we have shown that the conservation of the EM helicity can be used as a probe of pure-multipolar spectral regimes, particularly, of dipolar nature, arising well beyond its presumed spectral region. This intriguing finding shows that the dipolar behavior is not necessarily limited to small particles, where the point-dipole approximation works correctly [32]. From our results, it is straightforward to note

(a) Optimum forward
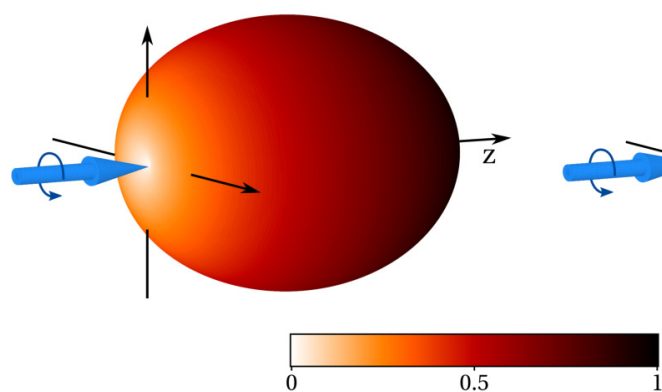

FIG. 4. Scattering radiation patterns at the first Kerker condition. (a) Optimum forward light scattering condition. (b) Scattering radiation patterns arising from Refs. [12,13], i.e., a $90 \mathrm{~nm} \mathrm{GaAs} \mathrm{sphere} \mathrm{at}$ $\lambda=775 \mathrm{~nm}$ and a $75 \mathrm{~nm} \mathrm{Si}$ sphere at $\lambda=660 \mathrm{~nm}$, respectively. 
that electric and magnetic dipolar optical forces [33-36], radiation pressure [37,38], light transport phenomena [39,40], and the novel concept of anapole modes [41], originally derived for small nanoparticles, can be extended to larger HRI dipolar spheres. Moreover, the novel dipolar regions where the EM helicity is preserved can be used to enhance the sensitivity of circular dichroism spectroscopy of chiral particles $[42,43]$. This could drive to experiments beyond the actual physical picture, which is mostly restricted to small HRI materials that present a relatively weak scattering efficiency at the first Kerker condition [44,45]. Finally, we have proved that the optimum forward light scattering condition, originally derived for a specific nanoparticle, is satisfied for an infinite number of materials at a fixed $x$ size parameter, in contrast to previous interpretations [20,21]. We believe that our results open new perspectives in the study of the light scattered by dielectric Mie spheres, including new possible applications of HRI particles as building blocks in all-dielectric optics and photonic devices.

This research was supported by the Basque Government (Project No. PI-2016-1-0041 and Ph.D. fellowship PRE2018-2-0252) and by the Spanish MINECO and MICINN and European Regional Development Fund (ERDF) Projects No. FIS2015-69295-C3-3-P, No. FIS2017-91413-EXP, No. FIS2017-82804-P, and No. PGC2018-095777-B-C21 and Ph.D. fellowship FPU15/03566. X.Z.-P. acknowledges funding from the European Union's Horizon 2020 research and innovation program under the Marie Sklodowska-Curie Grant Agreement No. 795838. J.J.S. and J.O.T. acknowledge support from Project No. PID2019-109905GA-C22 of the Spanish Ministerio de Ciencia, Innovación y Universidades (MICIU) and Gipuzkaoko Foru Aldundia Project No. OF23/2019 (ES).
[1] G. Mie, Ann. Phys. (Leipzig) 330, 377 (1908).

[2] M. Kerker, D.-S. Wang, and C. Giles, J. Opt. Soc. Am. A 73, 765 (1983).

[3] I. Fernandez-Corbaton, X. Zambrana-Puyalto, N. Tischler, X. Vidal, M. L. Juan, and G. Molina-Terriza, Phys. Rev. Lett. 111, 060401 (2013).

[4] M. Calkin, Am. J. Phys 33, 958 (1965).

[5] A. I. Kuznetsov, A. E. Miroshnichenko, M. L. Brongersma, Y. S. Kivshar, and B. Luk'yanchuk, Science 354, aag2472 (2016).

[6] A. García-Etxarri, R. Gómez-Medina, L. S. Froufe-Pérez, C. López, L. Chantada, F. Scheffold, J. Aizpurua, M. NietoVesperinas, and J. J. Sáenz, Opt. Express 19, 4815 (2011).

[7] M. Nieto-Vesperinas, R. Gomez-Medina, and J. J. Saenz, J. Opt. Soc. Am. A 28, 54 (2011).

[8] X. Zambrana-Puyalto, I. Fernandez-Corbaton, M. Juan, X. Vidal, and G. Molina-Terriza, Opt. Lett. 38, 1857 (2013).

[9] J. Olmos-Trigo, C. Sanz-Fernández, F. S. Bergeret, and J. J. Sáenz, Opt. Lett. 44, 1762 (2019).

[10] S. Nechayev, J. S. Eismann, G. Leuchs, and P. Banzer, Phys. Rev. B 99, 075155 (2019).

[11] J.-M. Geffrin, B. García-Cámara, R. Gómez-Medina, P. Albella, L. S. Froufe-Pérez, C. Eyraud, A. Litman, R. Vaillon, F. González, M. Nieto-Vesperinas, J. J. Sáenz, and F. Moreno, Nat. Commun. 3, 1171 (2012).

[12] S. Person, M. Jain, Z. Lapin, J. J. Sáenz, G. Wicks, and L. Novotny, Nano Lett. 13, 1806 (2013).

[13] Y. H. Fu, A. I. Kuznetsov, A. E. Miroshnichenko, Y. F. Yu, and B. Luk'yanchuk, Nat. Commun. 4, 1527 (2013).

[14] H. Barhom, A. A. Machnev, R. E. Noskov, A. Goncharenko, E. Gurvitz, A. S. Timin, V. A. Shkoldin, S. V. Koniakhin, O. Y. Koval, M. V. Zyuzin, et al., Nano Lett. 19, 7062 (2019).

[15] J. Jang, H. Jeong, G. Hu, C.-W. Qiu, K. T. Nam, and J. Rho, Adv. Opt. Mater. 7, 1970016 (2019).

[16] X. Zambrana-Puyalto and N. Bonod, Nanoscale 8, 10441 (2016).

[17] J. S. Eismann, M. Neugebauer, and P. Banzer, Optica 5, 954 (2018).
[18] J. Feis, D. Beutel, J. Köpfler, X. Garcia-Santiago, C. Rockstuhl, M. Wegener, and I. Fernandez-Corbaton, Phys. Rev. Lett. 124, 033201 (2020).

[19] F. Reyes Gómez, O. N. Oliveira Jr., P. Albella, and J. R. MejíaSalazar, Phys. Rev. B 101, 155403 (2020).

[20] Y. Zhang, M. Nieto-Vesperinas, and J. J. Sáenz, J. Opt. 17, 105612 (2015).

[21] B. S. Luk'yanchuk, N. V. Voshchinnikov, R. PaniaguaDomínguez, and A. I. Kuznetsov, ACS Photonics 2, 993 (2015).

[22] H. C. Hulst and H. C. van de Hulst, Light Scattering by Small Particles (Courier Corporation, 1981).

[23] J. Olmos-Trigo, C. Sanz-Fernández, D. R. Abujetas, J. LasaAlonso, N. de Sousa, A. García-Etxarri, J. A. Sánchez-Gil, G. Molina-Terriza, and J. J. Sáenz, Phys. Rev. Lett. 125, 073205 (2020).

[24] G. N. Watson, A Treatise on the Theory of Bessel Functions (Cambridge University Press, Cambridge, 1995).

[25] X. Zambrana-Puyalto, X. Vidal, M. L. Juan, and G. MolinaTerriza, Opt. Express 21, 17520 (2013).

[26] J. Olmos-Trigo, C. Sanz-Fernández, A. García-Etxarri, G. Molina-Terriza, F. S. Bergeret, and J. J. Sáenz, Phys. Rev. A 99, 013852 (2019).

[27] J. Olmos-Trigo, M. Meléndez, R. Delgado-Buscalioni, and J. J. Sáenz, Opt. Express 27, 16384 (2019).

[28] J. D. Jackson, Classical Electrodynamics (John Wiley \& Sons, New York, 1999).

[29] J. Olmos-Trigo, C. Sanz-Fernández, D. R. Abujetas, A. GarcíaEtxarri, G. Molina-Terriza, J. A. Sánchez-Gil, F. S. Bergeret, and J. J. Sáenz, J. Appl. Phys. 126, 033104 (2019).

[30] D. E. Aspnes and A. A. Studna, Phys. Rev. B 27, 985 (1983).

[31] M. I. Abdelrahman, C. Rockstuhl, and I. Fernandez-Corbaton, Sci. Rep. 7, 2608 (2017).

[32] P. C. Chaumet and M. Nieto-Vesperinas, Opt. Lett. 25, 1065 (2000).

[33] S. Albaladejo, M. I. Marqués, M. Laroche, and J. J. Sáenz, Phys. Rev. Lett. 102, 113602 (2009).

[34] A. Novitsky, C.-W. Qiu, and H. Wang, Phys. Rev. Lett. 107, 203601 (2011). 
[35] O. M. Maragò, P. H. Jones, P. G. Gucciardi, G. Volpe, and A. C. Ferrari, Nat. Nanotechnol. 8, 807 (2013).

[36] X. Xu, M. Nieto-Vesperinas, C.-W. Qiu, X. Liu, D. Gao, Y. Zhang, and B. Li, Laser Photonics Rev. 14, 1900265 (2020).

[37] P. H. Jones, O. M. Maragò, and G. Volpe, Optical Tweezers: Principles and Applications (Cambridge University Press, Cambridge, 2015).

[38] A. Y. Bekshaev, K. Y. Bliokh, and F. Nori, Phys. Rev. X 5, 011039 (2015).

[39] R. Gómez-Medina, L. S. Froufe-Pérez, M. Yépez, F. Scheffold, M. Nieto-Vesperinas, and J. J. Sáenz, Phys. Rev. A 85, 035802 (2012).

[40] J. Olmos-Trigo, D. R. Abujetas, C. Sanz-Fernández, J. A. Sánchez-Gil, and J. J. Sáenz, Phys. Rev. Res. 2, 013225 (2020).
[41] B. Luk'yanchuk, R. Paniagua-Domínguez, A. I. Kuznetsov, A. E. Miroshnichenko, and Y. S. Kivshar, Phys. Rev. A 95, 063820 (2017).

[42] M. Decker, M. Klein, M. Wegener, and S. Linden, Opt. Lett. 32, 856 (2007).

[43] M. Schäferling, D. Dregely, M. Hentschel, and H. Giessen, Phys. Rev. X 2, 031010 (2012).

[44] I. Fernandez-Corbaton, M. Fruhnert, and C. Rockstuhl, ACS Photonics 2, 376 (2015)

[45] M. L. Solomon, J. Hu, M. Lawrence, A. García-Etxarri, and J. A. Dionne, ACS Photonics 6, 43 (2018).

Correction: Support information for the first and last authors was missing from the Acknowledgment section and has been inserted. 